\title{
A52 THE ROLE OF THE WNT INHIBITOR SCLEROSTIN IN RHEUMATOID ARTHRITIS
}

C Wehmeyer, A Stratis, T Pap, B Dankbar Institute of Experimental Musculoskeletal Medicine - IEMM, University Hospital Muenster, Muenster, Germany

\subsection{6/ard.2010.129593n}

Introduction Joints affected by rheumatoid arthritis (RA) display signs of bone resorption and usually lack signs of repair which contributes to rapid and progressive loss of joint structures. Sclerostin is a potent inhibitor of osteoblast development by antagonising the Wnt/ $\beta$-catenin signalling pathway, a key regulator of bone turnover. Since it has been shown that sclerostin is expressed under inflammatory conditions, we analysed the role of sclerostin in bone destruction in RA.

Methods Sclerostin expression in vivo was determined by immunohistochemistry and western blot analysis in human synovial tissue of patients with RA and in the hind paws of tumour necrosis factor $\alpha$ transgenic (hTNFtg) mice which develop an RA-like destructive arthritis. In vitro, expression and regulation of sclerostin was analysed in cultured osteoarthritis (OA) and RA synovial fibroblasts, macrophages and in vitro differentiated osteoblasts and osteoclasts by real-time PCR and western blot analysis. Osteoclast differentiation was verified by tartrate-resistant acid phosphatase staining and osteoblast differentiation by analysis of osteocalcin and alkaline phosphatase expression.

Results Immunohistochemistry and western blot analysis revealed considerable overexpression of sclerostin in the synovial tissues of patients with RA compared with those with OA. Likewise, staining of ankle joint sections of hTNFtg mice revealed a high expression of sclerostin in the synovial tissue, especially in the infiltrating pannus, whereas synovial tissue of wild-type mice showed no staining. In vitro, constitutive expression of sclerostin was detected in differentiated osteoblasts, but expression of sclerostin could be induced also in human RA synovial fibroblasts by TNF $\alpha$.

Conclusions The disease-dependent synovial expression of sclerostin in human RA and arthritic mice suggests an important role of sclerostin in rheumatoid joint destruction. We hypothesise that inflammatory cytokines promote the imbalance between bone resorption and formation by affecting regulatory molecules of the Wnt pathway such as sclerostin. Targeting a pathway able to counter-regulate this imbalance of bone turnover may therefore be a key strategy in preventing structural damage in arthritis. 